\title{
THE SYMMETRIC NORMAL DERIVATIVE OF A SUBHARMONIC FUNCTION II
}

\author{
BENT FUGLEDE
}

\section{Introduction.}

In a previous paper [2] the author has proved that an arbitrary subharmonic function defined in a plane domain $\Omega$ possesses a symmetric normal derivative almost everywhere along any given line segment contained in $\Omega$. It was stated without proof that the analogous theorem holds for any twice differentiable curve in $\Omega$ instead of a line, and similarly in higher dimensions, where the curve should be replaced by a hypersurface.

It is the purpose of the present paper to supply a complete proof of this extension to curves in the plane and to $(n-1)$-dimensional manifolds in the $n$-dimensional Euclidean space $R^{n}$. Quite delicate-though elementary-estimates are required in order to accomplish the proof. The presentation is independent of the previous paper and is fairly selfcontained, the principal tools beyond classical analysis being the elements of integration theory and the Riesz representation theorem for subharmonic functions.

The symmetric normal derivative of a function $f=f(x)$ of $x \in R^{n}$ is defined at a point $x$ of an $(n-1)$-dimensional oriented smooth manifold $\Sigma$ by

$$
S f(x)=\lim _{s \rightarrow 0} \frac{f\left(x_{s}\right)-f\left(x_{-s}\right)}{2 s}
$$

provided this limit exists. Here $x_{s}=x+s N(x)$, where $N(x)$ denotes the positive unit normal to $\Sigma$ at $x$. Actually, we shall adopt in the present paper a somewhat more general definition of a symmetric normal derivative $S f$, which we obtain by replacing the normals, given parametrically by $s \rightarrow x_{s}=x+s N(x)$, by a smooth family of curves perpendicular to $\Sigma$ and determined by parametric representations $s \rightarrow x_{s}$. This generalization involves no complications in the proof of the results concerning subharmonic functions.

Among known smoothness properties of general subharmonic functions

Received November 31, 1962.

Math. Scand. $12-9$ 
we recall Evans' theorem (which will, however, not be used in the present paper), according to which a subharmonic function $f$ is absolutely continuous along almost every straight line in a prescribed direction (Evans [1, p. 233f.]). This implies the existence of the partial derivatives $\partial f / \partial x_{i}$ almost everywhere in the domain of $f .{ }^{1}$ In particular, the usual normal derivative exists almost everywhere on almost every hyperplane of a given direction. On a prescribed hyperplane, however, the norma derivative - unlike the symmetric normal derivative-need not exist anywhere, as we see from the example where the hyperplane is determined by $x_{1}=0$ and where $f(x)=\left|x_{1}\right|$.

The content of the present paper may be summarized as follows. In $\S 1$ we specify the requirements concerning the manifold $\Sigma$ and the family of curves $\Gamma_{x}$, which may take the place of the straight normals to $\Sigma$. Moreover, we formulate the results in Theorem 1 and in three propositions. In $\S 2$ we prepare the proof and reduce the matter to establishing a certain property of boundedness. (These steps are similar to the corresponding parts of the proof given in [2] for the special case $n=2$, $\Sigma=$ a line segment.) The verification of this boundedness condition takes up the following three sections, the crucial estimates being obtained in $\S 5$. The hypothesis that the curves $\Gamma_{x}$ be perpendicular to the manifold $\Sigma$ is used in Lemma $2, \S 5$.

We refer to the previous paper [2] for certain applications of the results obtained in the present paper to the structural characterization of subharmonic and $\delta$-subharmonic functions. (The proofs of these further results are given in [2] only for the two-dimensional case $n=2$, but the extension to higher dimensions is immediate.)

\section{Formulation of the results.}

The Euclidean $n$-dimensional space $R^{n}$ will be viewed as a vector space with the scalar product $\langle x, y\rangle$ and the norm $|x|=\langle x, x\rangle^{\frac{1}{2}}$, in terms of which the distance between $x$ and $y$ is written as $|x-y|$.

Let $\Sigma$ denote an oriented $(n-1)$-dimensional manifold of class $C^{2}$ imbedded in $R^{n}$. The surface measure on $\Sigma$ is denoted by $\sigma$ and the positive unit normal to $\Sigma$ at a point $x \in \Sigma$ by $N=N(x)$, where $|N(x)|=1$. For each point $x \in \Sigma$ let $\Gamma_{x}$ denote a curve in $R^{n}$ passing through $x$, defined as a mapping $s \rightarrow x_{s}$ of some interval on the real line $R$ into $R^{n}$. We suppose that $x_{s}$, considered as a function of the two variables $x \in \Sigma$ and $s \in R$, is defined in some open set $D \subset \Sigma \times R$ containing $\Sigma \times\{0\}$, and that the following two conditions are fulfilled: 
A. The mappings $(x, s) \rightarrow x_{s}$ and $(x, s) \rightarrow \partial x_{s} / \partial s$ of $D$ into $R^{n}$ are both of class $C^{1}(D)$.

B. $x_{s}=x$ and $\partial x_{s} / \partial s=N(x)$ for $s=0$ and any point $x \in \Sigma$.

Under these assumptions we say that the mapping $(x, s) \rightarrow x_{s}$ of $D$ into $R^{n}$ defines a normal family of curves $\Gamma_{x}$ with respect to $\Sigma$. The interpretation of the conditions $\mathrm{A}$ and $\mathrm{B}$ in terms of a local parametric representation of $\Sigma$ will be given in $\S 3$. It follows from A that the parametric representation $\Gamma_{x}: s \rightarrow x_{s}$ of each curve is of class $C^{2}$, and from B that the curve $\Gamma_{x}$ is perpendicular to $\Sigma$ at the point $x$ and oriented in accordance with the positive unit normal $N(x)$ to $\Sigma$ at $x$. Note that the parameter $s$ is not required to coincide with the are length $l$ from $x$ to $x_{s}$ along $\Gamma_{x}$, but it follows from $B$ that

$$
\frac{d l}{d s}=\left|\frac{\partial x_{s}}{\partial s}\right|=1 \quad \text { for } s=0 .
$$

It is easily shown that an $(n-1)$-dimensional manifold $\Sigma \subset R^{n}$ must necessarily be of class $C^{2}$ if there shall exist a normal family of curves with respect to $\Sigma$ in the sense specified above. In fact, it follows from $\mathrm{A}$ and $\mathrm{B}$ that $N(x)$ must be of class $C^{1}$ as a function of $x \in \Sigma$. - Conversely, if $\Sigma$ is an oriented $(n-1)$-dimensional manifold of class $C^{2}$ in $R^{n}$, then the positive normals to $\Sigma$, given parametrically by the mappings $s \rightarrow x_{s}=x+s N(x)$, obviously constitute a normal family of curves with respect to $\Sigma$. Note, however, that this mapping $(x, s) \rightarrow x_{s}$ is not of class $C^{2}$ unless $N(x)$ is of class $C^{2}$, that is, $\Sigma$ is of class $C^{3}$. It is for this reason that we have chosen to assume merely that the mappings $(x, s) \rightarrow x_{s}$ and $(x, s) \rightarrow \partial x_{s} / \partial s$ be of class $C^{1}$ - which just suffices for the proof of the subsequent results - instead of requiring $x_{s}$ to be of class $C^{2}$.

Returning to the general case of a normal family of curves $\Gamma_{x}: s \rightarrow x_{s}$ with respect to the $(n-1)$-dimensional manifold $\Sigma \subset R^{n}$, we consider a function $f$ with values in $\bar{R}=[-\infty,+\infty]$, defined in some open set $\Omega \subset R^{n}$ containing $\Sigma$. The symmetric normal derivative of $f$ with respect to the normal family of curves $\Gamma_{x}: s \rightarrow x_{s}$ is defined by

$$
S f(x)=\lim _{s \rightarrow 0} \frac{f\left(x_{s}\right)-f\left(x_{-s}\right)}{2 s}
$$

at any point $x \in \Sigma$ for which this limit exists in $\bar{R}$. (In particular, for all sufficiently small values of $|s|>0, f\left(x_{s}\right)$ and $f\left(x_{-s}\right)$ should not both be infinite in the same sense.) Obviously, it suffices to consider values $s>0$. - If $f$ happens to possess a total differential at some point $x \in \Sigma$, then clearly $S f$ exists at that point and coincides with the usual normal derivative. 
TheURem 1. Let $f=f(x)$ denote a function which is subharmonic in an open set $\Omega \subset R^{n}$, but otherwise arbitrary. Let $\Sigma$ denote an oriented $(n-1)$ dimensional manifold of class $C^{2}$ imbedded in $\Omega, \sigma$ the surface measure on $\Sigma$, and $N=N(x)$ the positive unit normal to $\Sigma$. Then, for any normal family of curves $\Gamma_{x}: s \rightarrow x_{s}$ with respect to $\Sigma$, the symmetric normal derivative (1) of $f$ exists at $\sigma$-almost every point $x \in \Sigma$ and determines a locally $\sigma$-integrable function Sf on $\Sigma$, the equivalence class of which is independent of the particular normal family of curves.

Proposition 1. Under the assumptions of the above theorem, there corresponds to any compact subset $\Sigma^{*}$ of $\Sigma$ and to any normal family of curves with respect to $\Sigma$ a number $\delta>0$ such that $f\left(x_{s}\right)$ is defined, finite, and continuous ${ }^{2}$ with respect to $s \in[-\delta, \delta]$ for $\sigma$-almost every $x \in \Sigma^{*}$, and such that moreover

$$
\begin{gathered}
\int_{\Sigma^{*}} \sup _{-\delta \leqq s \leqq \delta}\left|f\left(x_{s}\right)\right| d \sigma(x)<\infty, \\
\int_{\Sigma^{*}} \sup _{0<s \leqq \delta} \frac{\left|f\left(x_{s}\right)-f\left(x_{-s}\right)\right|}{2 s} d \sigma(x)<\infty .
\end{gathered}
$$

Corollary. The limit relation (1) holds not only pointwise almost everywhere in $\Sigma$, but also in the sense of local mean convergence of order 1. Explicitly,

$$
\lim _{s \rightarrow 0} \int_{\Sigma^{*}}\left|\frac{f\left(x_{s}\right)-f\left(x_{-s}\right)}{2 s}-S f(x)\right| d \sigma(x)=0
$$

for every compact set $\Sigma^{*} \subset \Sigma$.

We express this property by saying that the symmetric normal derivative exists in the mean of order 1 on compact parts of $\Sigma$. A similar statement applies to the limit relation $f\left(x_{s}\right) \rightarrow f\left(x_{t}\right)$ for $s \rightarrow t$ in $[-\delta, \delta]$ expressing the continuity of $f\left(x_{s}\right)$. The corollary follows immediately from Theorem 1 and Proposition 1 by application of Lebesgue's theorem on dominated convergence.

Propostion 2. If the subharmonic function $f$ has the form of a potential of a negative measure $-\mu, \mu \geqq 0$, on $R^{n}$, that is, if

$$
f(x)=\left\{\begin{aligned}
-\int|x-y|^{2-n} d \mu(y) & \text { for } n \geqq 3 \\
\int \log |x-y| d \mu(y) & \text { for } n=2,
\end{aligned}\right.
$$


then Sf may be determined $\sigma$-almost everywhere on $\Sigma$ by differentiation under the integral sign with respect to $x$ in the direction of the positive normal to $\Sigma$ as follows: ${ }^{3}$

$$
S f(x)=(n-2) \int \frac{\langle x-y, N(x)\rangle}{|x-y|^{n}} d \mu(y)=(n-2) \int \frac{\cos \theta}{r^{n-1}} d \mu,
$$

the integrals on the right being absolutely convergent for $\sigma$-almost every point $x \in \Sigma$. Moreover, for any compact set $\Sigma^{*} \subset \Sigma$, the following interchange of integrations is permissible:

$$
\begin{aligned}
\int_{\Sigma^{*}} S f(x) d \sigma(x) & =(n-2) \int d \mu(y) \int_{\Sigma^{*}} \frac{\langle x-y, N(x)\rangle}{|x-y|^{n}} d \sigma(x) \\
& =(n-2) \int d \mu \int_{\Sigma^{*}} \frac{\cos \theta}{r^{n-1}} d \sigma .
\end{aligned}
$$

Here $r=|x-y|$ is the distance between the point $x \in \Sigma$ and the point $y \in R^{n}$, and $\theta$ is the angle between $x-y$ and the positive normal to $\Sigma$ at $x$.

Returning to the case of a general subharmonic function $f$ defined in an open set $\Omega \subset R^{n}$, we denote by $\mu \geqq 0$ the measure associated with $f$ in the manner described in the Riesz representation theorem (F. Riesz [4]). (Equivalently, $\mu$ is determined by Poisson's formula ${ }^{3}$

$$
\Delta f=(n-2) \omega_{n} \mu,
$$

interpreted in the sense of the theory of distributions. Here $\omega_{n}$ denotes the total surface measure of the unit sphere in $R^{n}$.) Suppose now that the $C^{2}$-manifold $\Sigma \subset \Omega$ is the boundary of some bounded open set $G \subset \Omega$, and that the positive unit normal $N=N(x)$ to $\Sigma$ points into $\complement \bar{G}$ for every $x \in \Sigma$. Then we have the following result generalizing the classical formula $\int_{\Sigma}(\partial f / \partial N) d \sigma=\int_{G} \Delta f d x$, which holds if $f$ is smooth.

Proposition 3. Let $f$ be subharmonic in $\Omega, \mu \geqq 0$ the associated measure, and $G$ a bounded open subset of $\Omega$ with smooth boundary $\Sigma \subset \Omega$ as specified above. Then ${ }^{3}$

$$
\int_{\Sigma} S f(x) d \sigma(x)=(n-2) \omega_{n}\left(\mu(G)+\frac{1}{2} \mu(\Sigma)\right) .
$$

\section{Reduction of the proof to a certain boundedness condition.}

It is easily shown that any manifold imbedded in a Euclidean space is representable as the union of a denumerable family of compact subsets 
of the manifold. For this reason it is sufficient, in proving Theorem 1, to verify the existence of the limit (1) for $\sigma$-almost every point of $\Sigma^{*}$ and to show that $S f$, considered on $\Sigma^{*}$, is $\sigma$-integrable and $\sigma$-essentially independent of the choice of the normal family of curves $\Gamma_{x}$. Here $\Sigma^{*}$ denotes an arbitrary compact subset of $\Sigma$.

The measure $\mu \geqq 0$ associated with a subharmonic function $f$ in $\Omega$ in the manner described in the Riesz representation theorem is characterized by the following property. For any bounded open set $\Omega^{*}$ with closure $\overline{\Omega^{*}} \subset \Omega$ we have the decomposition

$$
f(x)=f^{*}(x)+h(x), \quad x \in \Omega^{*},
$$

where $h$ is harmonic in $\Omega^{*}$, and where $f^{*}$ denotes the potential of $-\mu^{*}$ as defined by the right hand side of (4), $\S 1$, with $\mu$ replaced by $\mu^{*}$. Here $\mu^{*}$ denotes the trace of $\mu$ on $\overline{\Omega^{*}}$ obtained by cancelling the masses outside $\overline{\Omega^{*}}$. We choose $\Omega^{*}$ so as to contain the prescribed compact subset $\Sigma^{*}$ of $\Sigma$ mentioned in Proposition 1. By virtue of Borel's covering theorem applied to the compact set $\Sigma^{*} \times\{0\} \subset D$, there is a number $d^{*}>0$ such that $x_{s}$ is defined (that is, $(x, s) \in D$ ) and belongs to $\Omega^{*}$ for all $(x, s) \in \Sigma^{*} \times\left[-d^{*}, d^{*}\right]$. If we can find a number $\delta>0$ such that Proposition 1 holds with $f$ replaced by $f^{*}$, and such that $S f^{*}$ exists almost everywhere on $\Sigma^{*}$ and its equivalence class is independent of the particular choice of the normal family of curves $\Gamma_{x}$, then the same statements will follow for the given function $f$. In fact, the term $h$, which is harmonic in $\Omega^{*}$, will be of no effect in these matters provided we choose $\delta<d^{*}$.

In the proof of Proposition 2 we may likewise suppose without loss of generality that the measure $\mu$ is (bounded and) of compact support since in any case the distant masses, e.g. the masses outside $\overline{\Omega^{*}}$, contribute to the potential (4) only by some function which is harmonic in $\Omega^{*}$, and for which the differentiation under the integral sign in (5) as well as the interchange of integrations in (6) is permissible. - Note that the independence of the equivalence class of $S f$ from the choice of the normal family of curves is implied by (5).

Summing up, we have now reduced the proof of Theorem 1 and Propositions 1 and 2 to establishing the following three statements, in which $f$ is defined in $R^{n}$ by (4) with $\mu \geqq 0$ of compact support, and $\Sigma^{*}$ denotes a compact subset of the manifold $\Sigma$ :

i) There is a number $\delta>0$ with the properties asserted in Proposition 1 .

ii) The limit relation ${ }^{3}$ 


$$
\lim _{s \rightarrow 0} \frac{f\left(x_{s}\right)-f\left(x_{-s}\right)}{2 s}=(n-2) \int \frac{\langle x-y, N(x)\rangle}{|x-y|^{n}} d \mu(y)
$$

holds for $\sigma$-almost every point $x \in \Sigma^{*}$.

iii) The formula (6) holds.

Finally, we observe that Proposition 3 likewise follows from these statements. Applying the Riesz representation theorem, and choosing $\Omega^{*}$ so as to contain the closure $\bar{G}$ of the given set $G$, we write again $f=f^{*}+h$ in $\Omega^{*}$, where $f^{*}$ is determined by the right hand side of (4) with $\mu$ replaced by the trace $\mu^{*}$ of $\mu$ on $\overline{\Omega^{*}}$. Since $h$ is harmonic in $\Omega^{*}$, we have $\int_{\Sigma}(\partial h / \partial N) d \sigma=\int_{G} \Delta h d x=0$, and hence, according to statement iii),

$$
\int_{\Sigma} S f d \sigma=\int_{\Sigma} S f^{*} d \sigma=(n-2) \int_{\Omega^{*}} d \mu(y) \int_{\Sigma} \frac{\langle x-y, N(x)\rangle}{|x-y|^{n}} d \sigma(x) .
$$

This implies the identity stated in Proposition 3 when it is observed that, by a classical formula,

$$
\frac{1}{\omega_{n}} \int_{\Sigma} \frac{\langle x-y, N(x)\rangle}{|x-y|^{n}} d \sigma(x)= \begin{cases}1 & \text { for } y \in G \\ \frac{1}{2} & \text { for } y \in \Sigma \\ 0 & \text { for } y \in \mathcal{G} .\end{cases}
$$

We begin the verification of the statements i), ii), and iii), to which all the results announced in $\S 1$ have now been reduced, by choosing a number $d>0$ with the property that $x_{s}$ is defined (that is, $(x, s) \in D$ ) for every pair $(x, s) \in \Sigma^{*} \times[-d, d]$. Replacing $x$ by $x_{s}$ in the kernel entering on the right of (4), we obtain the following "deformed kernel"

$$
K_{s}(x, y)= \begin{cases}-\left|x_{s}-y\right|^{2-n} & \text { for } n \geqq 3 \\ \log \left|x_{s}-y\right| & \text { for } n=2,\end{cases}
$$

where $x \in \Sigma^{*}, y \in R^{n}, s \in[-d, d]$. For fixed $(x, y), K_{s}(x, y)$ is continuous as a function of $s$ with values in $\bar{R}$. The corresponding symmetric difference quotient is denoted by

$$
L_{s}(x, y)=\frac{K_{s}(x, y)-K_{-s}(x, y)}{2 s}, \quad 0<s<d .
$$

For $s \rightarrow 0$ (and $x \neq y), L_{s}(x, y)$ converges pointwise to ${ }^{3}$

$$
L_{0}(x, y)=\left.\frac{\partial}{\partial s} K_{s}(x, y)\right|_{s=0}=(n-2) \frac{\langle x-y, N(x)\rangle}{|x-y|^{n}} .
$$


For any number $\delta>0, \delta<d$, we introduce the majorant

$$
M_{\delta}(x, y)=\sup _{-\delta \leqq \delta \leqq \delta}\left|K_{s}(x, y)\right|+\sup _{0<s \leqq \delta}\left|L_{s}(x, y)\right| .
$$

(Note that $M_{\delta}(x, x)=+\infty$ because $K_{0}(x, x)=-\infty$ ). According to Lebesgue's theorem on dominated convergence, the following passages to the limit under the integral sign:

$$
f\left(x_{s}\right)=\int K_{s}(x, y) d \mu(y) \rightarrow \int K_{t}(x, y) d \mu(y)=f\left(x_{t}\right)
$$

as $s \rightarrow t$ in $[-\delta, \delta]$; and

$$
\frac{f\left(x_{s}\right)-f\left(x_{-s}\right)}{2 s}=\int L_{s}(x, y) d \mu(y) \rightarrow \int L_{0}(x, y) d \mu(y)
$$

as $s \rightarrow 0$, are both permissible and lead to finite limits $f\left(x_{t}\right)$ and $S f(x)$, respectively, at any point $x \in \Sigma^{*}$ for which the expression

is finite. - Writing

$$
\Psi_{\delta}(x)=\int M_{\delta}(x, y) d \mu(y)
$$

$$
\Phi_{\delta}(y)=\int_{\Sigma^{*}} M_{\delta}(x, y) d \sigma(x), \quad y \in R^{n},
$$

we have by Fubini's theorem

$$
\int_{\Sigma^{*}} \Psi_{\delta}(x) d \sigma(x)=\int \Phi_{\delta}(y) d \mu(y) .
$$

Hence, if we can show that $\Phi_{\delta}(y)$ is bounded as a function of $y$, at least on bounded subsets of $R^{n}$, then the integral on the right of (11) is finite because $\mu$ is bounded and of compact support. Consequently, $\Psi_{\delta}$ will be integrable over $\Sigma^{*}$ and hence finite $\sigma$-almost everywhere on $\Sigma^{*}$. From this latter property will then follow that $f\left(x_{s}\right)$ is finite and - in view of (9) - continuous as a function of $s \in[-\delta, \delta]$ for $\sigma$-almost every $x \in \Sigma^{*}$; and the limit relation (10) will hold, likewise for $\sigma$-almost every $x \in \Sigma^{*}$. The integrability of $\Psi_{\delta}$ over $\Sigma^{*}$ will imply the domination properties (2) and (3). In fact, it follows from (4), (7), and (8) that

and

$$
\left|f\left(x_{s}\right)\right|=\left|\int K_{s}(x, y) d \mu(y)\right| \leqq \Psi_{\delta}(x)
$$

$$
(2 s)^{-1}\left|f\left(x_{s}\right)-f\left(x_{-s}\right)\right|=\left|\int L_{s}(x, y) d \mu(y)\right| \leqq \Psi_{\delta}(x)
$$


for $-\delta \leqq s \leqq \delta$ and $0<s \leqq \delta$, respectively. Finally, the interchange of integrations (6) is permissible according to Fubini's theorem because

$$
\begin{aligned}
(n-2) \int d \mu(y) \int_{\Sigma^{*}} \frac{|\langle x-y, N(x)\rangle|}{|x-y|^{n}} d \sigma(x) & =\int d \mu(y) \int_{\Sigma^{*}}\left|L_{0}(x, y)\right| d \sigma(x) \\
& \leqq \int \Phi_{\delta}(y) d \mu(y)<\infty .
\end{aligned}
$$

In this manner we have reduced the proof of the statements i), ii), and iii), $\S 2$ (and hence the proof of Theorem 1 and Propositions 1, 2, and 3, $\S 1)$ to showing that there is a number $\delta>0$ such that $\Phi_{\delta}(y)$ is bounded as a function of $y$ on every bounded subset of $R^{n}$. Here $\Sigma^{*}$ was meant to denote an arbitrary prescribed compact subset of the manifold $\Sigma$. It follows, however, from Borel's covering theorem that it is enough to prove that there corresponds to each point $x^{*} \in \Sigma$ a number $\delta>0$ and a neighbourhood $\Sigma^{*}$ of $x^{*}$ in $\Sigma$ such that $\Sigma^{*} \times[-\delta, \delta] \subset D$ (the domain of the mapping $\left.(x, s) \rightarrow x_{s}\right)$, and such that the function

$$
\Phi_{\delta}(y)=\int_{\Sigma^{*}} M_{\delta}(x, y) d \sigma(x)
$$

is bounded on every bounded subset of $R^{n}$.

\section{Construction of $\delta$ and $\Sigma^{*}$.}

According to the definition of an $(n-1)$-dimensional manifold of class $C^{2}$ imbedded in $R^{n}$, there corresponds to the given point $x^{*} \in \Sigma$ an open neighbourhood $\Sigma_{0} \subset \Sigma$ of $x^{*}$ which is representable as the image of a suitable open set $U_{0} \subset R^{n-1}$ under a regular $C^{2}$-homeomorphism

$$
x=\xi(u)
$$

of $U_{0}$ into $R^{n}$. We may suppose that $\xi(0)=x^{*}$. The Cartesian coordinates $x_{i}=\xi_{i}(u)=\xi_{i}\left(u_{1}, \ldots, u_{n-1}\right)$ are functions of class $C^{2}\left(U_{0}\right)$, and the Jacobians

$$
J_{i}=(-1)^{n-i} \frac{\partial\left(x_{1}, \ldots, x_{i-1}, x_{i+1}, \ldots, x_{n}\right)}{\partial\left(u_{1}, \ldots, u_{n-1}\right)}
$$

do not vanish simultaneously at any point $u \in U_{0}$. The vector $J=J(u)=$ $\left(J_{1}, \ldots, J_{n}\right)$ is proportional to the unit normal $N$ at the point $x=\xi(u)$ of $\Sigma_{0}$. The hypothesis that $\Sigma$ be oriented means that the parametric representation $x=\xi(u)$ of $\Sigma_{0}$ may be so chosen that

$$
J=+|J| N .
$$


Here

$$
|J|=\left(J_{1}^{2}+\ldots+J_{n}^{2}\right)^{\frac{1}{2}}=d \sigma / d u .
$$

The mapping $(x, s) \rightarrow x_{s}$ of an open set $D \subset \Sigma \times R$ into $R^{n}$, which serves to define the normal family of curves $\Gamma_{x}$ with respect to $\Sigma$, may now be expressed (for $x \in \Sigma_{0}$ ) in terms of the parametric representation $x=\xi(u)$ of $\Sigma_{0}$ as follows:

$$
(u, s) \rightarrow \eta(u, s)\left(=x_{s} \text { with } x=\xi(u)\right) .
$$

Here $(u, s)$ ranges over the set $W_{0}$ of pairs $(u, s)$ such that $u \in U_{0}$ and $(\xi(u), s) \in D$, the domain of the mapping $(x, s) \rightarrow x_{s}$. Clearly, $W_{0}$ is an open neighbourhood of the pair $(u, s)=(0,0)$. - If we write, for brevity,

$$
\partial \eta(u, s) / \partial s=\zeta(u, s),
$$

then the properties $\mathrm{A}$ and $\mathrm{B}$ of the mapping $(x, s) \rightarrow x_{s}$ (cf. $\S 1$ ) are reflected as follows (for $x \in \Sigma_{0}$ ):

a. The mappings $(u, s) \rightarrow \eta(u, s)$ and $(u, s) \rightarrow \zeta(u, s)$ of $W_{0}$ into $R^{n}$ are both of class $C^{1}\left(W_{0}\right)$.

b. $\eta(u, 0)=\xi(u)$ and $\zeta(u, 0)=N(\xi(u))$ for every $u \in U_{0}$.

It follows from these two properties that the Jacobian $I=I(u, s)$ of the mapping $(u, s)=\left(u_{1}, \ldots, u_{n-1}, s\right) \rightarrow \eta(u, s)$ is equal to $|J(u)|=d \sigma / d u$ for $s=0$. We obtain, in fact, from (12), (13), and (14)

$$
I(u, 0)=\sum_{i=1}^{n} J_{i}(u) \zeta_{i}(u, 0)=\langle J, N\rangle=|J|=d \sigma / d u>0 .
$$

We shall only need this result in so far as it implies that $I(0,0) \neq 0$. Since $(u, s) \rightarrow \eta(u, s)$ is a $C^{1}$-mapping of an open neighbourhood $W_{0} \subset$ $U_{0} \times R$ of the point $(u, s)=(0,0)$, and since the Jacobian $I(u, s)$ is non zero at that point, there is an open neighbourhood $W_{1} \subset W_{0}$ of $(0,0)$ such that the restriction of $\eta$ to $W_{1}$ is one-to-one and of Jacobian $I(u, s) \neq 0$. In other words, the restriction of $\eta$ to $W_{1}$ is a regular $C^{1}$ homeomorphism, and so is therefore the inverse of this restriction.

Now let $B_{a}=\left\{u \in R^{n-1}:|u| \leqq a\right\}$ denote the closed ball of radius $a$ about the origin in $R^{n-1}$. We choose $a>0$ so small that the cylinder

$$
C_{a}=B_{a} \times[-a, a]=\left\{(u, s) \in R^{n-1} \times R:|u| \leqq a,|s| \leqq a\right\}
$$

is contained in $W_{1}$. Since $C_{a}$ is compact, so is its image $\eta\left(C_{a}\right)$, and hence $\eta$ and $\eta^{-1}$ are Lipschitz mappings between $C_{a}$ and $\eta\left(C_{a}\right)$. This means that there is a constant $k$ such that the inequalities ${ }^{4}$

$$
k^{-1}(|u-v|+|s-t|) \leqq|\eta(u, s)-\eta(v, t)| \leqq k(|u-v|+|s-t|)
$$


hold for $(u, s)$ and $(v, t)$ in the cylinder $C_{a}=B_{a} \times[-a, a]$. - Since $\zeta=$ $\partial \eta / \partial s$ is likewise of class $C^{1}$ (according to property $a$ ), there is a constant $c$ such that

$$
|\zeta(u, s)-\zeta(v, t)| \leqq c(|u-v|+|s-t|)
$$

for $(u, s)$ and $(v, t)$ in $C_{a}$. Note also that $d \sigma / d u=|J(u)|$ is bounded in $B_{a}$.

We proceed to show that $\Phi_{\delta}(y)=\int_{\Sigma^{*}} M_{\delta}(x, y) d \sigma(x)$ is a bounded function of $y$ on every bounded subset of $R^{n}$ provided $\delta<a$ and $\Sigma^{*}=\xi\left(B_{\delta}\right)$, the compact neighbourhood of $x^{*}$ in $\Sigma$ obtained as the image of the ball $B_{\delta}=\left\{u \in R^{n-1}:|u| \leqq \delta\right\}$ under the parametric representation $x=\xi(u)=$ $\eta(u, 0)$ of the original neighbourhood $\Sigma_{0}$ of $x^{*}$ in $\Sigma$. We distinguish the two cases $y \in \bigcap \eta\left(C_{a}\right)$ and $y \in \eta\left(C_{a}\right)$.

\section{Boundedness of $\Phi_{\delta}$ on bounded subsets of $\left\lceil\eta\left(C_{a}\right)\right.$.}

In order to show that the function $\Phi_{\delta}(y)=\int_{\xi\left(B_{\delta}\right)} M_{\delta}(x, y) d \sigma(x)$ of $y \in R^{n}$ is bounded for bounded $y$ not in $\eta\left(C_{a}\right)$, we recall that the cylinder $C_{\delta}=$ $B_{\delta} \times[-\delta, \delta]$ is compact and contained in the interior of $C_{a}$. Hence $\eta\left(C_{\delta}\right)$ is a compact subset of the interior of $\eta\left(C_{a}\right)$. This implies the existence of a constant $b>0$ such that $|z-y| \geqq b$ for $z \in \eta\left(C_{\delta}\right), y \in \complement \eta\left(C_{a}\right)$. In particular,

$$
\left|x_{s}-y\right| \geqq b \quad \text { for } \quad x \in \xi\left(B_{\delta}\right), s \in[-\delta, \delta], y \in\left\lceil\eta\left(C_{a}\right)\right.
$$

because $x=\xi(u)=\eta(u, 0)$ for some $u \in B_{\delta}$; and so $x_{s}=\eta(u, s)$, where $(u, s) \in B_{\delta} \times[-\delta, \delta]=C_{\delta}$. - It is now clear that, for $n \geqq 3$, the deformed kernel $K_{s}(x, y)$ defined in $\S 2$ is uniformly bounded for $x \in \xi\left(B_{\delta}\right)$, $s \in[-\delta, \delta], y \in \complement \eta\left(C_{a}\right)$. For $n=2$ we obtain uniform boundedness of $K_{s}(x, y)$ for $y$ in a bounded subset of $\complement \eta\left(C_{a}\right)$. Consequently, the expression

$$
\int_{\xi\left(B_{\delta}\right)} \sup _{|s| \leqq \delta}\left|K_{s}(x, y)\right| d \sigma(x)
$$

is bounded as a function of $y$ in any bounded subset of $\left\lceil\eta\left(C_{a}\right)\right.$.

In order to estimate the symmetric difference quotient $L_{s}(x, y)$ defined in (7), $\S 2$, we shall make use of the following elementary inequalities valid for any two vectors $p$ and $q$ in $R^{n}$ of length $|p| \geqq b,|q| \geqq b$, where $b>0$ denotes some given number:

$$
\begin{cases}\left.|| p\right|^{2-n}-|q|^{2-n}|\leqq(n-2)| p-q \mid b^{1-n} & \text { for } n \geqq 3 \\ |\log | p|-\log | q|| \leqq|p-q| b^{-1} & \text { for } n=2 .\end{cases}
$$

(Actually, these inequalities subsist even with $|p-q|$ on the right replaced by ||$p|-| q||$, and in this stronger form they follow e.g. by application of the mean-value theorem in one variable.) 
Applying (18) to $L_{s}(x, y)$ with $p=x_{s}-y, q=x_{-s}-y$, and $b$ equal to the constant introduced a while ago, cf. (17), we obtain for $x \in \xi\left(B_{\delta}\right)$, $0<s \leqq \delta$, and $y \in\left\lceil\eta\left(C_{a}\right),{ }^{3}\right.$

$$
\left|L_{s}(x, y)\right| \leqq(2 s)^{-1}(n-2)\left|x_{s}-x_{-s}\right| b^{1-n} \leqq(n-2) k b^{1-n},
$$

because $\left|x_{s}-x_{-s}\right|=|\eta(u, s)-\eta(u,-s)| \leqq 2 k s$ on account of the Lipschitz conditions (15). It follows that the expression

$$
\int_{\xi\left(B_{\delta}\right)} \sup _{0<s \leqq \delta}\left|L_{s}(x, y)\right| d \sigma(x)
$$

is bounded for $y \in\left\lceil\eta\left(C_{a}\right)\right.$.

Summing up, we have established the boundedness of $\Phi_{\delta}(y)=$ $\int_{\Sigma^{*}} M_{\delta}(x, y) d \sigma(x)$ on bounded subsets of $\left\lceil\eta\left(C_{a}\right)\right.$. Here $\delta$ was any number $<a$, and $\Sigma^{*}=\xi\left(B_{\delta}\right)$ was the image of the ball $B_{\delta}$ under the parametric representation $x=\xi(u)$ of $\Sigma_{0}$.

\section{Boundedness of $\Phi_{\delta}(y)$ for $y \in \eta\left(C_{a}\right)$.}

In the proof that $\Phi_{\delta}(y)$ is bounded as a function of $y \in \eta\left(C_{a}\right)$, it turns out that we may just as well take $\delta=a, \Sigma^{*}=\eta\left(B_{a}\right)$ instead of $\delta<a$, $\Sigma^{*}=\eta\left(B_{\delta}\right)$. In other words, we propose to show that the expression

$$
\int_{\xi\left(B_{a}\right)} M_{\delta}(x, y) d \sigma(x)
$$

is bounded for $y \in \eta\left(C_{a}\right)$ provided $\delta \leqq a$. Thus, from now on, the three relevant points $x, x_{s}$, and $y$ of $R^{n}$ all belong to the image $\eta\left(C_{a}\right)$ of the cylinder $C_{a}=B_{a} \times[-a, a]$ under the Lipschitz mapping $(u, s) \rightarrow \eta(u, s)$.

Accordingly, we shall write

$$
x=\eta(u, 0), \quad x_{s}=\eta(u, s), \quad y=\eta(v, t),
$$

where $u, v \in B_{a}, s, t \in[-a, a]$. For brevity, we put

$$
\begin{cases}r=|x-y|=|\eta(u, 0)-\eta(v, t)|, & \varrho=|u-v|+|t| \\ r_{s}=\left|x_{s}-y\right|=|\eta(u, s)-\eta(v, t)|, & \varrho_{s}=|u-v|+|s-t| .\end{cases}
$$

Then

$$
\begin{aligned}
K_{s} & =K_{s}(x, y)= \begin{cases}-r_{s}{ }^{2-n} & \text { for } n \geqq 3 \\
\log r_{s} & \text { for } n=2,\end{cases} \\
L_{s} & =L_{s}(x, y)= \begin{cases}(2 s)^{-1}\left(r_{-s}{ }^{2-n}-r_{s}{ }^{2-n}\right) & \text { for } n \geqq 3 \\
(2 s)^{-1} \log \left(r_{s} / r_{-s}\right) & \text { for } n=2\end{cases}
\end{aligned}
$$


According to the Lipschitz conditions (15), we have

$$
k^{-1} \varrho \leqq r \leqq k \varrho, \quad k^{-1} \varrho_{s} \leqq r_{s} \leqq k \varrho_{s} .
$$

Our task is to estimate $K_{s}$ and $L_{s}$ (expressed in terms of $u$, $v$, and $t$ instead of $x$ and $y$ ) uniformly with respect to $s$ (and to $u$, $v$, and $t$ ). We formulate the result to be obtained as follows.

Fundamental estimates. For $x=\eta(u, 0)$ and $y=\eta(v, t)$ we have, writing $\varrho=|u-v|+|t|$,

$$
\begin{aligned}
& K_{s}(x, y)= \begin{cases}O\left(|u-v|^{2-n}\right) & \text { for } n \geqq 3 \\
O\left(\log \left(1+|u-v|^{-1}\right)\right) & \text { for } n=2,\end{cases} \\
& L_{s}(x, y)= \begin{cases}O\left(\left(1+|t| \varrho^{-2}\right)|u-v|^{2-n}\right) & \text { for } n \geqq 3 \\
O\left(\left(1+|t| \varrho^{-2}\right) \log \left(2+2|t||u-v|^{-1}\right)\right) & \text { for } n=2\end{cases}
\end{aligned}
$$

Here, and elsewhere in the sequel, we use Landau's symbol $O(\ldots)$ uniformly with respect to $u, v, s$, and $t$ for $u, v \in B_{a}$ and $s, t \in[-a, a]$. The estimate (21) for $K_{s}$ follows for $n \geqq 3$ from the following inequalities derived from (19) and (20):

$$
r_{s} \geqq k^{-1} \varrho_{s} \geqq k^{-1}|u-v| .
$$

For $n=2$ we have, since $\varrho_{s} \leqq|u|+|v|+|s|+|t| \leqq 4 a$,

and hence

$$
k^{-1}|u-v| \leqq k^{-1} \varrho_{s} \leqq r_{s} \leqq k \varrho_{s} \leqq 4 k a,
$$

$$
-\log k-\log \left(|u-v|^{-1}\right) \leqq \log r_{s} \leqq \log (4 k a) .
$$

Observing that $|u-v| \leqq 2 a$, so that $\log \left(1+|u-v|^{-1}\right)$ is bounded from below by a positive constant, we obtain $1=O\left(\log \left(1+|u-v|^{-1}\right)\right)$, and the stated estimate of $K_{s}$ for $n=2$ follows.

The estimate (22) of $L_{s}$ is considerably more delicate. We shall need the following two lemmas, of which the first is quite simple and similar to the inequalities (18), §4. We may suppose $s>0$.

Lemma 1. If $r_{s} \geqq b$ and $r_{-s} \geqq b$ for some $s>0$ and $b>0$, then ${ }^{3}$

$$
\left|L_{s}\right| \leqq(n-2)(4 s)^{-1}\left|r_{-s}^{2}-r_{s}^{2}\right| b^{-n} \text {. }
$$

Proof. Apply the mean-value theorem to the functions $\lambda^{1-n / 2}$ (for $n \geqq 3$ ), and $\log \left(\lambda^{\frac{1}{2}}\right)$ (for $n=2$ ) of a real variable $\lambda$ over the interval between $r_{-s}^{2}$ and $r_{s}^{2}$, and observe that $\lambda \geqq b^{2}$ throughout this interval.

Lemma 2. For $u, v \in B_{a}, 0<s \leqq a$, and $-a \leqq t \leqq a$, we have the uniform estimate

$$
(2 s)^{-1}\left(r_{-s}{ }^{2}-r_{s}^{2}\right)=2 t+O(\varrho+s)^{2} .
$$


Proof. We have

$$
r_{-s}^{2}-r_{s}^{2}=\left|y-x_{-s}\right|^{2}-\left|y-x_{s}\right|^{2}=\left\langle 2 y-x_{s}-x_{-s}, x_{s}-x_{-s}\right\rangle .
$$

Expanding $x_{s}=\eta(u, s)$ from $(u, 0)$ by means of Taylor's formula, we obtain on account of properties a and $b, \S 3$,

$$
x_{s}=\eta(u, 0)+s \zeta(u, 0)+O\left(s^{2}\right)=x+s N(x)+O\left(s^{2}\right)
$$

uniformly for $u \in B_{a}, s \in[-a, a]$. Inserting this expression for $x_{s}$ and the corresponding expression for $x_{-s}$ in the identity above, we obtain, taking from now on $s>0$,

$$
\begin{aligned}
r_{-s}^{2}-r_{s}^{2} & =\left\langle 2 y-2 x+O\left(s^{2}\right), 2 s N(x)+O\left(s^{2}\right)\right\rangle \\
& =4 s\langle y-x, N(x)\rangle+O\left(s^{2} \varrho+s^{3}\right),
\end{aligned}
$$

uniformly for $u, v \in B_{a}, 0<s \leqq a$, and $-a \leqq t \leqq a$. We have used here that $|x-y|=r \leqq k \varrho$ (cf. (19) and (20)) and that $|N(x)|=1$.

In order to estimate $\langle y-x, N(x)\rangle$, we evaluate $y=\eta(v, t)$, again under observation of properties a and $b, \S 3$. We obtain

$$
\begin{gathered}
y=\eta(v, t)=\eta(v, 0)+t \zeta(v, 0)+O\left(t^{2}\right), \\
\eta(v, 0)=\eta(u, 0)+\sum_{i=1}^{n-1}\left(v_{i}-u_{i}\right) \partial \xi(u) / \partial u_{i}+O\left(|v-u|^{2}\right), \\
\zeta(v, 0)=\zeta(u, 0)+O(|v-u|)=N(x)+O(|v-u|) .
\end{gathered}
$$

Since the second term on the right of (25) is a vector in the tangent space to the manifold $\Sigma$ at the point $x=\xi(u)=\eta(u, 0)$, this term is perpendicular to the unit normal $N(x)$ at that point, ${ }^{5}$ and hence we obtain, subtracting $x=\eta(u, 0)$ from (24), inserting $\eta(v, 0)$ from (25), and projecting on $N(x)$,

$$
\langle y-x, N(x)\rangle=\left\langle t \zeta(v, 0)+O\left(t^{2}\right)+O\left(|v-u|^{2}\right), N(x)\right\rangle .
$$

Using (26), we obtain

$$
\begin{aligned}
\langle y-x, N(x)\rangle & =\left\langle t N(x)+O\left(|t||u-v|+|t|^{2}+|u-v|^{2}\right), N(x)\right\rangle \\
& =t+O(|t|+|u-v|)^{2}=t+O\left(\varrho^{2}\right) .
\end{aligned}
$$

Summing up, we infer from (23) and (27) the estimate

$$
\begin{aligned}
r_{-s}^{2}-r_{s}^{2} & =4 s t+O\left(s \varrho^{2}+s^{2} \varrho+s^{3}\right) \\
& =2 s\left(2 t+O(\varrho+s)^{2}\right) .
\end{aligned}
$$

This completes the proof of Lemma 2 .

We are now prepared to establish the fundamental estimate (22) of $L_{s}$. It is, however, necessary to distinguish three cases according to the 
relative magnitudes of $s, t$ and $\varrho=|u-v|+|t|$. It is assumed throughout that $u \in B_{a}, v \in B_{a}, 0<s \leqq a,-a \leqq t \leqq a$; and all estimates are uniform within this range. We may suppose throughout that $u \neq v$, and hence $\varrho \neq 0$.

The case $s \leqq \varrho / 2$. In this case we have from (19) and (20)

$$
r_{ \pm s} \geqq k^{-1} \varrho_{ \pm s} \geqq k^{-1}(\varrho-s) \geqq k^{-1} \varrho / 2
$$

and hence we may take $b=k^{-1} \varrho / 2$ in Lemma 1. Inserting $s \leqq \varrho / 2$ in the estimate given in Lemma 2, we obtain $(2 s)^{-1}\left(r_{-s}^{2}-r_{s}^{2}\right)=2 t+O\left(\varrho^{2}\right)$, and we conclude from Lemma 1 that

$$
\begin{aligned}
L_{s}(x, y)=O\left(\left(|t|+\varrho^{2}\right) \varrho^{-n}\right) & =O\left(\left(|t| \varrho^{-2}+1\right) \varrho^{2-n}\right) \\
& =O\left(\left(|t| \varrho^{-2}+1\right)|u-v|^{2-n}\right)
\end{aligned}
$$

because $\varrho \geqq|u-v|$. This leads to the estimates stated in (22) when it is observed, for $n=2$, that $1=O\left(\log \left(2+2|t||u-v|^{-1}\right)\right)$.

The case $s \geqq \varrho / 2 \geqq 2|t|$. Here we have

$$
r_{ \pm s} \geqq k^{-1} \varrho_{ \pm s}=k^{-1}(|u-v|+| \pm s-t|) \geqq k^{-1}(|u-v|+s / 2) .
$$

In fact, $| \pm s-t| \geqq s-|t| \geqq s / 2$ because $s \geqq 2|t|$. Applying Lemma 1 with $b=k^{-1}(|u-v|+s / 2)$, and inserting $\varrho \leqq 2 s$ in Lemma 2 , we obtain

$$
\begin{aligned}
L_{s}(x, y) & =O\left(\left(|t|+s^{2}\right)(|u-v|+s / 2)^{-n}\right) \\
& =O\left(\left(|t|+s^{2}\right)(|u-v|+s / 2)^{-2}|u-v|^{2-n}\right) \\
& =O\left(\left(|t||u-v|^{-2}+s^{2}(s / 2)^{-2}\right)|u-v|^{2-n}\right) \\
& =O\left(\left(|t| \varrho^{-2}+1\right)|u-v|^{2-n}\right)
\end{aligned}
$$

because $|u-v|=\varrho-|t| \geqq 3 \varrho / 4$ on account of the hypothesis $|t| \leqq \varrho / 4$. This implies, as above, the estimates stated in (22) as well for $n \geqq 3$ as for $n=2$.

The case $s \geqq \varrho / 2,2|t| \geqq \varrho / 2$. Suppose first $n \geqq 3$. Since

$$
r_{ \pm s} \geqq k^{-1} \varrho_{ \pm s} \geqq k^{-1}|u-v|,
$$

we obtain immediately by a very crude estimate

Inserting

$$
L_{s}(x, y)=(2 s)^{-1}\left(r_{-s}^{2-n}-r_{s}^{2-n}\right)=O\left(s^{-1}|u-v|^{2-n}\right) .
$$

$$
s^{-1} \leqq 2 \varrho^{-1}=2 \varrho \varrho^{-2} \leqq 8|t| \varrho^{-2}=O\left(1+|t| \varrho^{-2}\right),
$$

we get $L_{s}(x, y)=O\left(\left(1+|t| \varrho^{-2}\right)|u-v|^{2-n}\right)$, which is the estimate stated in (22) for $n \geqq 3$. 
For $n=2$ we have

and hence

$$
k^{-2} \varrho_{s} / \varrho_{-s} \leqq r_{s} / r_{-s} \leqq k^{2} \varrho_{s} / \varrho_{-s},
$$

$$
\log \left(r_{s} / r_{-s}\right)=\log \left(\varrho_{s} / \varrho_{-s}\right)+O(1) .
$$

It is easily verified that the ratio

$$
\frac{\varrho_{s}}{\varrho_{-s}}=\frac{|u-v|+|s-t|}{|u-v|+|s+t|}
$$

attains its maximal and minimal values (as a function of $s \in R$ ) at $s=-t$ and $s=t$, respectively. Consequently,

$$
\left|\log \left(\varrho_{s} / \varrho_{-s}\right)\right| \leqq \log \left(\varrho_{-t} / \varrho_{t}\right)=\log \left(1+2|t||u-v|^{-1}\right) .
$$

Combining (28), (29), and (30), we obtain the estimate stated in (22) for $n=2$ since $1=O\left(\log \left(2+2|t||u-v|^{-1}\right)\right.$.

Having thus verified the uniform estimates (21) and (22) for $K_{s}(x, y)$ and $L_{s}(x, y)$, we arrive at the following estimate for the majorant $M_{\delta}(x, y)$ introduced in (8), $\S 2$, the number $\delta$ being any positive number $\leqq a$ :

$$
M_{\delta}(x, y)= \begin{cases}O\left(\left(1+|t| \varrho^{-2}\right)|u-v|^{2-n}\right) & \text { for } n \geqq 3 \\ O\left(|t| \varrho^{-2} \log \left(2+2|t||u-v|^{-1}\right)+\log \left(1+|u-v|^{-1}\right)\right) & \text { for } n=2\end{cases}
$$

As before, $\varrho=|u-v|+|t|$. In deriving (31) from (21) and (22), we have used the estimate

$$
\begin{aligned}
\log \left(2+2|t||u-v|^{-1}\right) & =\log \left(|u-v|^{-1}\right)+\log (2|u-v|+2|t|) \\
& \leqq \log \left(1+|u-v|^{-1}\right)+\log (6 a) \\
& =O\left(\log \left(1+|u-v|^{-1}\right)\right)
\end{aligned}
$$

valid because $|t| \leqq a,|u-v| \leqq 2 a$, and hence $\log \left(1+|u-v|^{-1}\right)$ is bounded from below. The resulting estimate (31) of $M_{\delta}(x, y)$ has been shown to hold uniformly for $x \in \xi\left(B_{a}\right), y \in \eta\left(C_{a}\right)$, provided $\delta \leqq a$. Our task is to prove that

$$
\int_{\xi\left(B_{a}\right)} M_{\delta}(x, y) d \sigma(x)
$$

remains bounded for $y \in \eta\left(C_{a}\right)$. Since $d \sigma / d u$ is bounded for $u \in B_{a}$, it is sufficient to verify that the following integrals over the ball $B_{a}=$ $\left\{u \in R^{n-1}:|u| \leqq a\right\}$ are bounded functions of $(v, t)$ for $v \in B_{a}, t \in[-a, a]$. For $n \geqq 3$ the integrals in question are 


$$
\begin{aligned}
& I_{1}=\int_{B_{a}}|u-v|^{2-n} d u, \\
& I_{2}=\int_{B_{a}}|t| \varrho^{-2}|u-v|^{2-n} d u .
\end{aligned}
$$

For $n=2$ the ball $B_{a}$ reduces to the segment $[-a, a]$, and the integrals in question are

$$
\begin{aligned}
& I_{3}=\int_{-a}^{a} \log \left(1+|u-v|^{-1}\right) d u \\
& I_{4}=\int_{-a}^{a}|t| \varrho^{-2} \log \left(2+2|t||u-v|^{-1}\right) d u .
\end{aligned}
$$

In the integral $I_{1}$ we replace the ball $B_{a}$ by the ball of radius $2 a$ about $v$. This new domain of integration contains $B_{a}$ because $|v| \leqq a$. In $I_{2}$ we replace $B_{a}$ by the entire space $R^{n-1}$. Similarly, in $I_{3}$, we replace the interval $[-a, a]$ by the interval $[v-2 a, v+2 a]$, which contains it; and in $I_{4}$ we integrate instead over the entire real line. Denote the four integrals derived in this manner from $I_{1}, \ldots, I_{4}$ by $I_{1}{ }^{\prime}, \ldots, I_{4}{ }^{\prime}$. Since $\varrho=|u-v|+|t|$, the point of integration $u \in R^{n-1}$ enters only in the form of the distance $p=|u-v|$ from the point $v$. Moreover, in the four new integrals, the domains of integration have rotational symmetry with respect to $v$. Consequently, $I_{1}{ }^{\prime}, \ldots, I_{4}{ }^{\prime}$ reduce to simple integrals with respect to $p$, and we obtain, denoting by $\omega_{n-1}$ the total surface measure of the unit sphere in $R^{n-1}$,

$$
\begin{aligned}
I_{1} \leqq I_{1}^{\prime} & =\omega_{n-1} \int_{0}^{2 a} p^{2-n} p^{n-2} d p=2 a \omega_{n-1} \\
I_{2} \leqq I_{2}^{\prime} & =\omega_{n-1} \int_{0}^{\infty}|t|(p+|t|)^{-2} p^{2-n} p^{n-2} d p=\omega_{n-1} \\
I_{3} \leqq I_{3}^{\prime} & =2 \int_{0}^{2 a} \log \left(1+p^{-1}\right) d p<\infty \\
I_{4} \leqq I_{4}^{\prime} & =2 \int_{0}^{\infty}|t|(p+|t|)^{-2} \log \left(2+2|t| p^{-1}\right) d p \\
& =2 \int_{0}^{\infty}(q+1)^{-2} \log \left(2+2 q^{-1}\right) d q<\infty
\end{aligned}
$$

Math. Scand. $12-10$ 
where we have substituted $p=|t| q$ in the last integral (under the assumption $t \neq 0$ ). Having thus shown that $I_{1}{ }^{\prime}, \ldots, I_{4}{ }^{\prime}$ are finite constants, and hence that $I_{1}, \ldots, I_{4}$ are bounded functions of $(v, t)$ in the cylinder $C_{a}=B_{a} \times[-a, a]$, we conclude from (31) that $\Phi_{\delta}(y)=\int_{\xi\left(B_{a}\right)} M_{\delta}(x, y) d \sigma(x)$ is indeed bounded for $y=\eta(v, t) \in \eta\left(C_{a}\right)$. Combining this result with the result of $\S 4$, we have proved the assertion formulated at the end of $\S 2$, and hence completed the proof of Theorem 1 and Propositions 1, 2, and $3, \S 1$.

\section{Notes.}

1 Evans' theorem can be generalized as follows. Let $1<p<n /(n-1)$. Suppose $f$ is subharmonic in $\Omega$, and denote by $\Omega^{*}$ an open set of compact closure $\overline{\Omega^{*}} \subset \Omega$. Then $f$ belongs to the class of Beppo Levi functions denoted in [3] by $B L^{p}\left(\Omega^{*}\right)$. This means that $f$ is absolutely continuous along $p$-almost every rectifiable curve $\gamma \subset \Omega^{*}$, and the partial derivatives $\partial f / \partial x_{i}$ are of class $L^{p}\left(\Omega^{*}\right)$. The assertion that $f$ is absolutely continuous along p-almost every curve $\gamma \subset \Omega^{*}$ means by definition (cf. [3], p. 187) that there is a lower semicontinuous function $F \geqq 0$ of class $L^{p}\left(\Omega^{*}\right)$ such that $f$ is absolutely continuous along any rectifiable curve $\gamma \subset \Omega^{*}$ for which $\int_{\gamma} F(x) d s<\infty$ (with $s=$ arc length along $\gamma$ ). In the proof of this extension of Evans' theorem we may suppose, by virtue of the Riesz representation theorem, that $f$ is the potential of a negative measure - $\mu$ of compact support. The convolution $F(x)=|x|^{1-n} * \mu$ is obviously lower semicontinuous and of class $L^{p}$ on compact subsets of $R^{n}$ for any $p<n /(n-1)$ because the kernel $|x|^{1-n}$ has these properties. It is easily verified by application of Evans' method that $f$ is absolutely continuous along any rectifiable curve $\gamma \subset \Omega^{*}$ for which $\int_{\gamma} F^{\prime}(x) d s<\infty$.

2 This statement would hold for any smooth family of curves transversal to $\Sigma$. Actually, it can be shown that $f\left(x_{8}\right)$ is absolutely continuous as a function of $s \in[-\delta$, $\delta]$ for $\sigma$-almost every point $x \in \Sigma^{*}$. This follows easily from the generalization of Evans' theorem mentioned in the preceding note.

3 Here, and elsewhere in the present paper, the factor $n-2$ should be replaced by 1 in the case $n=2$.

4 It is slightly more convenient to use the distance $|u-v|+|s-t|$ in $R^{n-1} \times R$ instead of the Euclidean distance $\left(|u-v|^{2}+|s-t|^{2}\right)^{\frac{1}{2}}$ to which it is equivalent.

s It is at this step only that the hypothesis that the curves $\Gamma_{x}$ be perpendicular to $\Sigma$ (and not just transversal to $\Sigma$ ) is used effectively.

\section{REFERENCES}

1. G. C. Evans, On potentials of positive mass I, Trans. Amer. Math. Soc. 37 (1935), $226-253$.

2. B. Fuglede, The symmetric normal derivative of a subharmonic function I, Treizième congrès des mathématiciens scandinaves, Helsinki 1957 (1958), 90-101.

3. B. Fuglede, Extremal length and functional completion, Acta Math. 98 (1957), 171-219.

4. F. Riesz, Sur les fonctions subharmoniques et leur rapport à la théorie du potentiel II, Acta Math. 54 (1930), 321-360. 